Voix et Images

voixetimages

\title{
Critique et roman : des rapports dialogiques
}

\section{Jacques Pelletier}

Volume 15, numéro 3 (45), printemps 1990

\section{Gilbert La Rocque}

URI : https://id.erudit.org/iderudit/200860ar

DOI : https://doi.org/10.7202/200860ar

Aller au sommaire du numéro

Éditeur(s)

Université du Québec à Montréal

ISSN

0318-9201 (imprimé)

1705-933X (numérique)

Découvrir la revue

Citer cet article

Pelletier, J. (1990). Critique et roman : des rapports dialogiques. Voix et Images, 15(3), 443-448. https://doi.org/10.7202/200860ar d'utilisation que vous pouvez consulter en ligne.

https://apropos.erudit.org/fr/usagers/politique-dutilisation/ 


\section{Critique et roman: des rapports dialogiques}

\section{par Jacques Pelletier, Université du Québec à Montréal}

La critique, on le sait, constitue un maillon fort de la chaîne formant ce que l'on a convenu d'appeler, à la suite de Bourdieu, les instances de consécration et de légitimation du champ littéraire, chaîne que parcourt tout texte depuis sa production jusqu'à sa reconnaissance institutionnelle. C'est la critique sous ses diverses formes (journalistique, médiatique, académique, etc.) qui détermine en effet pour l'essentiel le destin d'une œuvre. Aussi n'est-il pas sans intérêt de connaître ce qu'elle retient dans le flot de la production et en vertu 
de quels critères elle opère, qu'elle en soit consciente ou non, une sélection.

Dans cette perspective on ne peut que se réjouir de l'initiative prise par Richard Hodgson et Ralph Sarkonak d'examiner comment la production romanesque récente (1960-1986) au Québec a été reçue par la critique. Réunissant dans un ouvrage collectif les travaux de collègues, eux-mêmes critiques, sur le discours d'accompagnement (qu'il soit positif ou négatif) pratiqué par le corps des lecteurs spécialisés que constituent les officiers de la critique, opérant ainsi en quelque sorte un travail de méta-critique, ils dressent un constat à la fois de l'état actuel de la critique québécoise et de la production romanesque que celle-ci prend sous sa coupe ${ }^{1}$.

Dans cette production, Hodgson et Sarkonak distinguent, pour des fins opératoires, cinq principaux courants: les romans dits du "texte national " caractérisant surtout les années 1960, le roman féministe surgissant et s'imposant durant les années 1970 , le roman postmoderne naissant au tournant des années 1980 mais dont on trouve des traits déjà dans les textes d'un Aquin ou d'un Ducharme notamment, enfin, renvoyant à des catégories davantage stylistiques qu'historiques, les romans de la parole, prolongeant une certaine tradition orale (à titre d'exemple, les chroniques du Plateau de Michel Tremblay), et ceux de l'écriture, influencés par le discours moderniste parisien (Tel Quel et apparentés). Cette classification, comme toute construction de cette nature, ne va pas sans opérer quelque réduction, quelque simplification, mais elle s'avère à mon sens pertinente et juste de manière générale en ce qu'elle permet de voir un certain ordre, une certaine logique dans le foisonnement et la prolifération en tous sens de la production romanesque de cette période.

Hodgson lui-même soumet une étude très éclairante de la critique réservée à l'œuvre de Jacques Godbout. Il montre que celle-ci a suscité deux grandes catégories de travaux: narratologiques et stylistiques d'une part, psychocritiques et sociologiques comme on pouvait s'y attendre d'autre part. Jusqu'ici, ce sont les analyses "idéologiques » de cette production qui se sont avérées les plus nombreuses et les plus conséquentes, confirmant l'intuition qu'on pouvait entretenir quant au statut de cette œuvre comme cristallisation exemplaire des préoccupations de toute une génération d'écrivains apparus dans le champ littéraire au milieu des années 1960, s'inscrivant peu ou prou dans le sillage de Parti pris. Hodgson conclut son bilan en signalant certaines zones encore inexplorées dans l'œuvre de Godbout, notamment la confrontation dans son écriture du français et de l'anglais, la lutte de ces langues dans le texte, sa "bilinguisation", à quoi il faudrait selon moi inclúre la prise en compte du "français de France" également en rapport dialectique avec le français "québécois" qui imprègne l'œuvre, ce conflit des langues exprimant 
par ailleurs à sa manière toute la complexité de la question nationale québécoise.

Grazia Merler; pour sa part, évoque les critiques consacrées aux Fous de Bassan d'Anne Hébert. La formulation de son étude est un peu "scolaire", mais sa démonstration est rigoureuse et elle est suivie d'une utile bibliographie.

On peut en dire tout autant de l'apport de Sarkonak concernant la production de Gérard'Bessette: le chercheur montre comment cette œuvre a inspiré des critiques relevant de diverses orientations (un peu comme celle de Godbout): stylistique, thématique, psychocritique surtout, ce questionnement étant en quelque sorte "appelé" par la nature même des écrits de l'auteur, lui-même impénitent psychocritique. Il s'agit encore là d'un travail bien conduit, en dépit du fait que Sarkonak privilégie peut-être trop l'ouvrage collectif, Lectures de Gérard Bessette, dirigé par Jean-Jacques Hamm² 2

Dans une section du livre intitulée "Positions critiques et nouvelles perspectives ", Janet Paterson s'intéresse à la post-modernité du roman et de la critique québécoise. Selon elle, une partie importante de la production romanesque relèverait de la post-modernité: elle invoque à titre d'exemples les œuvres d'Aquin, de Godbout, de Poulin qui s'inspireraient pour l'essentiel de cette esthétique, en plus de textes singuliers généralement perçus comme post-modernes par la critique: French Kiss de Brossard, la Vie en prose de Villemaire, etc. Cette classification, bien entendu, ne va pas de soi ni pour Aquin et Godbout généralement considérés comme brillants représentants du courant dit du "texte national ", ni pour Poulin dont l'œuvre présente des parentés structurelles avec le roman américain, à moins évidemment que celui-ci soit perçu comme post-moderne, à condition également d'isoler les traits formels, compositionnels des œuvres d'Aquin et de Godbout des thèmes qu'ils véhiculent. Paterson ouvre ainsi une stimulante discussion susceptible de provoquer une réévaluation, une perception nouvelle d'une partie considérable du corpus romanesque de la période. Par ailleurs, si on ne peut pas proprement parler d'une critique post-moderne, du moins peut-on signaler certaines études (de Caroline Bayard, de Ginette Michaud, de Janet Paterson elle-même) orientées vers la recherche de marques, de traces de la post-modernité et ouvrant ainsi une nouvelle perspective critique sur l'ensemble de la production romanesque québécoise.

Dans la même section, il faut également signaler l'excellent article de Réjean Beaudoin, bilan de la production éclatée des années 1980 réalisé à partir de ce qu'on en a dit: il réussit à présenter une vision d'ensemble de celle-ci, ce qui n'est pas rien, compte tenu qu'il ne dispose pas de la nécessaire distance historique.

Áutres contributions intéressantes: celle de Pierre Hébert sur la réception de Roch Carrier au Canada anglais et celle de Mary Jean 
Green sur la critique universitaire des romancières québécoises aux États-Unis.

Au-delà de l'évocation factuelle des analyses de l'œuvre de Carrier pratiquées par les anglophones, Hébert s'interroge sur la signification de cette réception: pourquoi cette production jouit-elle d'un tel engouement, qu'est-ce qui en elle l'autorise? Question d'autant plus pertinente que cette œuvre n'a pas reçu - encore - un accueil significatif de la critique québécoise, et singulièrement du milieu académique: pourquoi ne s'y est-on pas intéressé ici? Question qui nous interpelle, animateurs de revues universitaires que nous sommes.

Green, quant à elle, montre que les romancières québécoises font l'objet d'importantes études dans les universités américaines: c'est le cas notamment des œuvres de Marie-Claire Blais, Nicole Brossard, Anne Hébert, Jovette Marchessault et France Théoret qui sont abordées le plus généralement à partir de préoccupations féministes. Le bilan dressé par Green est succint mais précis, et accompagné, comme celui d'Hébert d'ailleurs, d'une bibliographie pertinente.

Dans l'ensemble, on le voit, les articles réunis dans cette publication en respectent l'orientation générale. Cependant quelques collaborateurs enfreignent, à des degrés divers, les normes et frontières du genre.

Ainsi Ben Z. Shek, s'il évoque fidèlement les analyses consacrées à l'Hiver de force de Réjean Ducharme, ne peut s'empêcher de polémiquer avec Renée Leduc-Park dont les interprétations lui apparaissent discutables, voire erronées; il fait de même à l'égard de Josianne Bornstein qui pratique selon lui une approche naïvement socio-critique et il "exécute" Claude Filteau pour avoir proposé une lecture dans une optique lacanienne qui reste bien abstraite et difficile à suivre pour les non-initiés (p. 55). Reste qu'il faut lui reconnaître le mérite d'avoir tenu compte des critiques formulées sur le roman de Ducharme.

Ce que ne fait guère Laurent Mailhot dans son étude de Volkswagen Blues de Jacques Poulin et autres «histoires américaines" du Québec. En réalité, il s'agit essentiellement d'une analyse de quelques romans québécois travaillés / traversés par un imaginaire "américain", finement conduite comme c'est le plus souvent le cas chez Mailhot, élégamment écrite comme toujours, mais qui passe allègrement à côté de la question. Si bien que j'ai été un peu surpris de la voir figurer au début de l'ouvrage, comme première application de la démarche indiquée par les animateurs du collectif: elle aurait mieux trouvé sa place dans la section consacrée aux "Positions critiques et nouvelles perspectives".

Le dossier est fermé par un article de Chantal de Grandpré sur la critique française du roman québécois. Enfin, il devrait s'agir de cela 
comme le suggère le titre de sa contribution. Dans les faits, il n'en est rien. Il s'agit plutôt d'un règlement de comptes avec Hubert Aquin, particulièrement vicieux, écrit par une plume chargée de fiel.

De Grandpré, par exemple, insinue qu'Aquin aurait été un étudiant paresseux, sinon peu doué, ne complétant qu'une année d'études durant un séjour de trois ans en France, qu'un personnage médiatique sachant orchestrer et publiciser ses gestes, si bien qu'il aurait créé luimême de toutes pièces les conditions de succès de Prochain Épisode au Québec. Le roman est ensuite disqualifié en tant que fausse fiction et fausse autobiographie - d'un faussaire invétéré donc - et les critiques françaises de Prochain Épisode sont évoquées à l'appui de ce jugement péremptoire.

Bien entendu, de Grandpré a le droit de penser ce qu'elle veut de cette œuvre et de son auteur. Est-elle fondée pour autant à recourir à la médisance, sinon à la calomnie, pour servir son propos? L'évocation du rapport des psychiatres sur l'engagement d'Aquin dans le F.L.Q. en 1964 (quel rapport? il n'en est fait mention ici que sur le mode allusif), de ses soi-disant difficultés à écrire Neige noire réduit de mauvaise foi à un scénario de film, de son impuissance à rendre à terme le projet d'Obombre; enfin du rôle qu'il aurait prétendument joué dans la fabrication des rumeurs contre Réjean Ducharme en 1966-1967, tout cela ne concerne guère, c'est le moins qu'on puisse dire, laccueil de Prochain Épisode à Paris.

La démonstration de Grandpré est particulièrement tordue dans l'analyse qu'elle soumet concernant les rumeurs contre Ducharme. Elle recourt alors à la bonne vieille tactique stalinienne de l'amalgame. Ainsi Aquin est à Paris lorsque le journal d'extrême-droite, Minute, lance ce qu'elle appelle la "seconde rumeur" contre Ducharme. Donc, Aquin, laisse-t-elle entendre, en est l'auteur. C'est ce que l'on appelle un procès par association: vous connaissez $\mathrm{X}$, celui-ci est d'extrême-droite, donc vous êtes d'extrême-droite (ainsi Aquin etc.): c'est avec ce genre de logique qu'on a pendu bien des innocents!

Pour les fins de sa démonstration, de Grandpré ne répugne à rien. Ainsi elle s'en prend de façon oblique à Jacques Allard à propos d'une hypothèse de celui-ci sur la mauvaise réception de Prochain Épisode à Paris, et de manière plus sournoise encore à René Lapierre dont elle écrit que son roman, l'Été Rébecca, a été reçu dans le silence, silence qui équivaut, comme de bien entendu, à une condamnation d'une entreprise de déréalisation qui n'a pourtant pas grand-chose à voir avec l'invention (p. 133). Cette hargne se porte assez curieusement, mais sûrement pas par l'effet du hasard, sur deux des principaux anïmateurs du projet ÉDAQ (Édition critique de l'œuvre d'Hubert Aquin) pour lequel de Grandpré a travaillé et où elle a notamment puisé les "informations " qu'elle utilise de manière malicieuse dans son texte: en clair, elle crache dans la soupe qui l'a nourrie, ce qui n'est pas très joli. 
Cette opération assez répugnante se fait sous le couvert d'un examen des critères littéraires qui auraient présidé aux jugements portés par la critique française sur la production romanesque québécoise. En cela, de Grandpré entend se démarquer de la perspective sociologique adoptée par Jaequeline Gerols dans le Roman québécois en France; prise de distance d'autant plus nécessaire que celleci, dans la conclusion de son ouvrage, écrit que si le public a boudé Hubert Aquin, c'est en grande partie parce que son œuvre n'a pas été portée à l'attention des literati susceptibles de l'apprécier, en raison du manque de "sérieux" littéraire de l'image de son éditeur auprès des critiques lettrés, à cette époque ${ }^{3}$. Explication d'ordre institutionnel beaucoup plus sérieuse, on l'admettra, que celle qui fait appel aux soi-disant défauts littéraires de l'œuvre.

Cette entreprise de dénigrement à l'endroit d'Aquin trouve ses fondements, on ne s'en surprendra pas, dans une attitude hautaine à l'égard non seulement de la littérature québécoise, mais de la communauté que celle-ci exprime. De Grandpré peut bien penser ce qu'elle veut du Québec, cracher sa bile tant qu'elle voudra, reste qu'on est en droit de s'interroger sur la pertinence de sa contribution à un ouvrage de haute tenue sur la production romanesque contemporaine et les discours critiques qui nous permettent souvent de poser sur celle-ci un regard neuf. Sur ce plan-là, on doit convenir que Hodgson et Sarkonak ont manqué de jugement et que de Grandpré a raté une belle occasion de se taire.

1 Richard Hodgson et Ralph Sarkonak (directeurs), le Roman québécois contemporain (1960-1986) devant la critique. Cuvres et critiques, Tübingen/ Paris, Gunter Narr/Sedes, 1989, 160 p.

2 J.J. Hamm (compilateur), Lectures de Gérard Bessette, Montréal, Québec/ Amérique, 1982 (Littérature d'Amérique).

3 Jacqueline Gerols, le Roman québécois en France, Montréal, Hurtubise HMH, 1984 p. 324 (Littérature). 